\title{
Focused Review: Efficacy of the Rector Spinae Plane Block
}

\author{
Hironobu Ueshima*, Hiroshi Otake \\ Department of Anesthesiology, Showa University Hospital, Tokyo, Japan \\ Email: *ueshimhi@yahoo.co.jp
}

How to cite this paper: Ueshima, $H$. and Otake, H. (2018) Focused Review: Efficacy of the Rector Spinae Plane Block. Open Journal of Anesthesiology, 8, 214-222. https://doi.org/10.4236/ojanes.2018.87022

Received: June 19, 2108

Accepted: July 22, 2018

Published: July 25, 2018

Copyright (C) 2018 by authors and Scientific Research Publishing Inc. This work is licensed under the Creative Commons Attribution International License (CC BY 4.0).

http://creativecommons.org/licenses/by/4.0/

\begin{abstract}
Since the original publication on the erector spinae plane (ESP) block in 2016, the technique of the ESP block has evolved significantly in the last few years. This review highlights recent developments in the technique for administering the ESP block and proposes directions for future research. Continuous efforts are being aimed at improving understanding regarding the administration of the ESP block. Current reports suggest that the ESP block provides effective analgesia in thoracic and abdominal sites in patients of all ages. However, no cohort studies or randomized controlled trials were performed in 2016 and 2017. The ESP block is an effective analgesic tool in a wide range of sites. However. We are uncertain how effective the ESP block is compared to other types of regional anesthesia. Therefore, more research on ESP blocks is required.
\end{abstract}

\section{Keywords}

Peripheral Nerve Block, Erector Spinae Plane, Thoracic and Abdominal Analgesic Tool

\section{Introduction}

The erector spinae plane (ESP) block was first described by Forero et al. [1]. Currently, the ESP block is performed as one of the pain management procedures for patients of all generation (newborns, infant, children, adolescents and adults) undergoing abdominal and thoracic surgeries [1]-[6].

In spite of the many publications on ESP blocks, there are still unclear aspects of the technique such as the spread of local anesthetic and mechanisms of action.

\section{Anatomy of the Thoracolumbar Fascia (Figure 1)}

To understand the ESP block better, we must first understand the anatomy of 


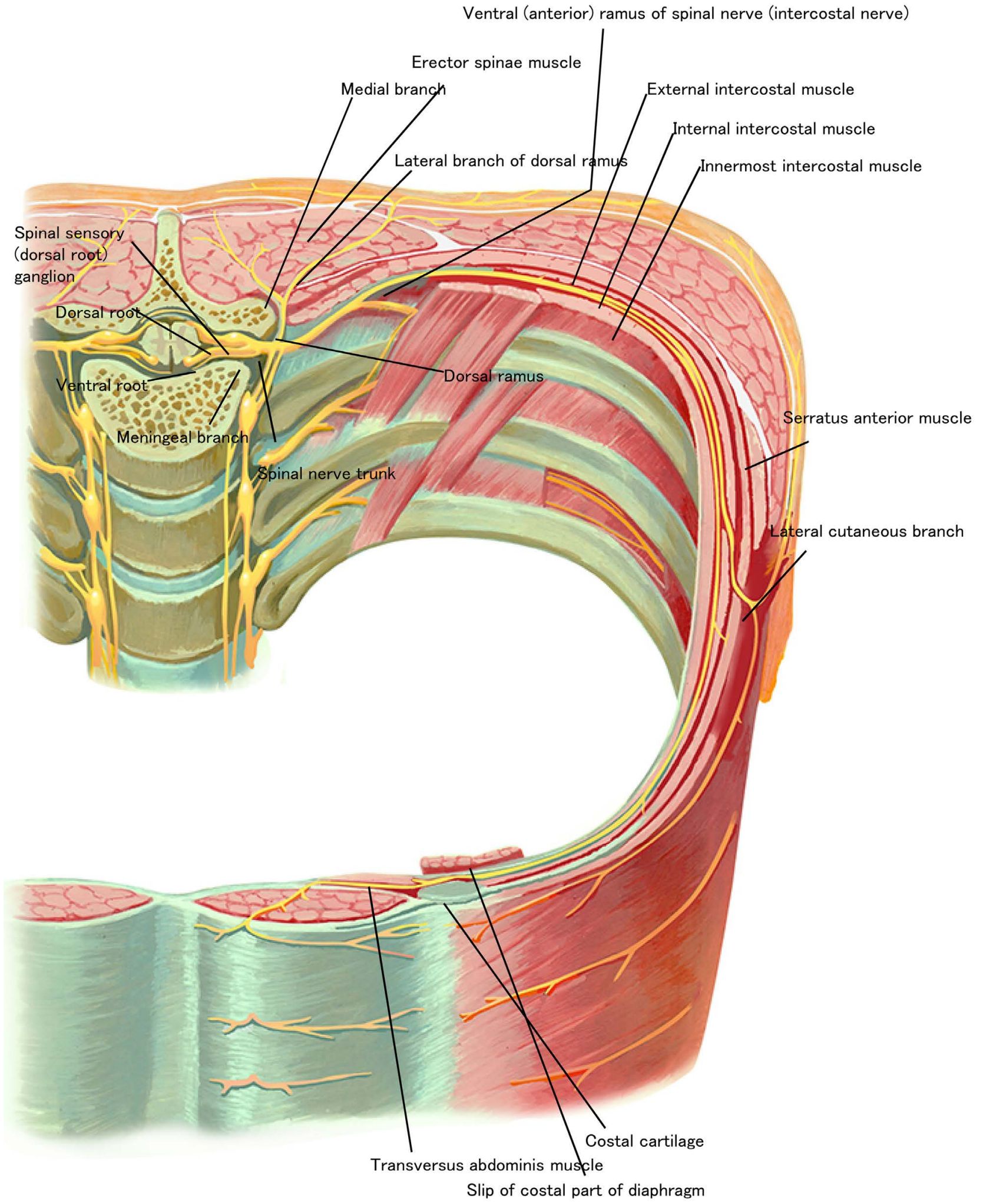

Figure 1. Anatomy of the thoracolumbar fascia (referred to Atlas of Human Anatomy 6th Edition).

the region, particulary, the thoracolumbar fascia [7].

At the upper thoracic site, the spinal nerve splits into the dorsal and ventral 
rami at their exit from the intervertebral foramen' exist. The dorsal ramus runs posteriorly through the costotransverse foramena and ascends into the erector spinae muscle. The thoracic dorsal ramus then divides into lateral and medical branches. The medial branch continues to ascend through the rhomboid major and trapezius muscles to superficial location before ending in a posterior cutaneous branch. The lateral cutaneous branch arises from the intercostal nerve next to the angle of the rib and this branch then ascends to a superficial location. The ventral ramus runs laterally, becoming the intercostal nerve, into a plane between the internal and innermost intercostal muscle on the inner aspect of the rib.

\section{Nomenclature (Figure 2)}

The first publication on the ESP block reports 2 different approaches (ESP 1 block and ESP 2 block) [1].

The ESP 1 block: Local anesthetic is injected into the interfascial plane between the rhomboid major and the erector spinae muscles.

The ESP 2 block: Local anesthetic is injected into the interfascial plane between the erector spinae muscle and the underlying transverse process.

Forero et al. currently recommended the ESP 2 block over the ESP 1 block. We could not perform the ESP 1 block at lower levels, because the rhomboid muscle has its inferior border at T6. The ESP 2 block below was expressed the ESP block.

\section{Techniques of ESP Block}

Although the first publication suggested the patient must be in a sitting position, the ESP block is currently performed in all position [1] [2] [3] [8]. A high-frequency linear ultrasound transducer is placed against the target vertebral level and moved in a cranio-caudal direction $3 \mathrm{~cm}$ lateral to the spinous process. The erector spinae muscle is then identified and a needle is advanced through the interfascial plane between the erector spinae and the underlying transverse process, following which the local anesthetic is administered into the space.

Although most studies mainly report on injections targeted at the transverse process, this method can miss the erector spinae sheath and prevent the spread of local anesthetic [9]. Compared to the transverse process, the sagittal process is clearer to spread of local anesthetic [10].

\section{Comparison of ESP Block with Retrolaminar Block}

ESP blocks and retrolaminar blocks are very similar peripheral nerve blocks in terms of anatomical target, spread of local anesthetic and analgesic effect [11] [12]. The anatomical site in ESP blocks is the transverse process, whereas in retrolaminar blocks, it is the lamina. In other words, only the insertion sites are different. 


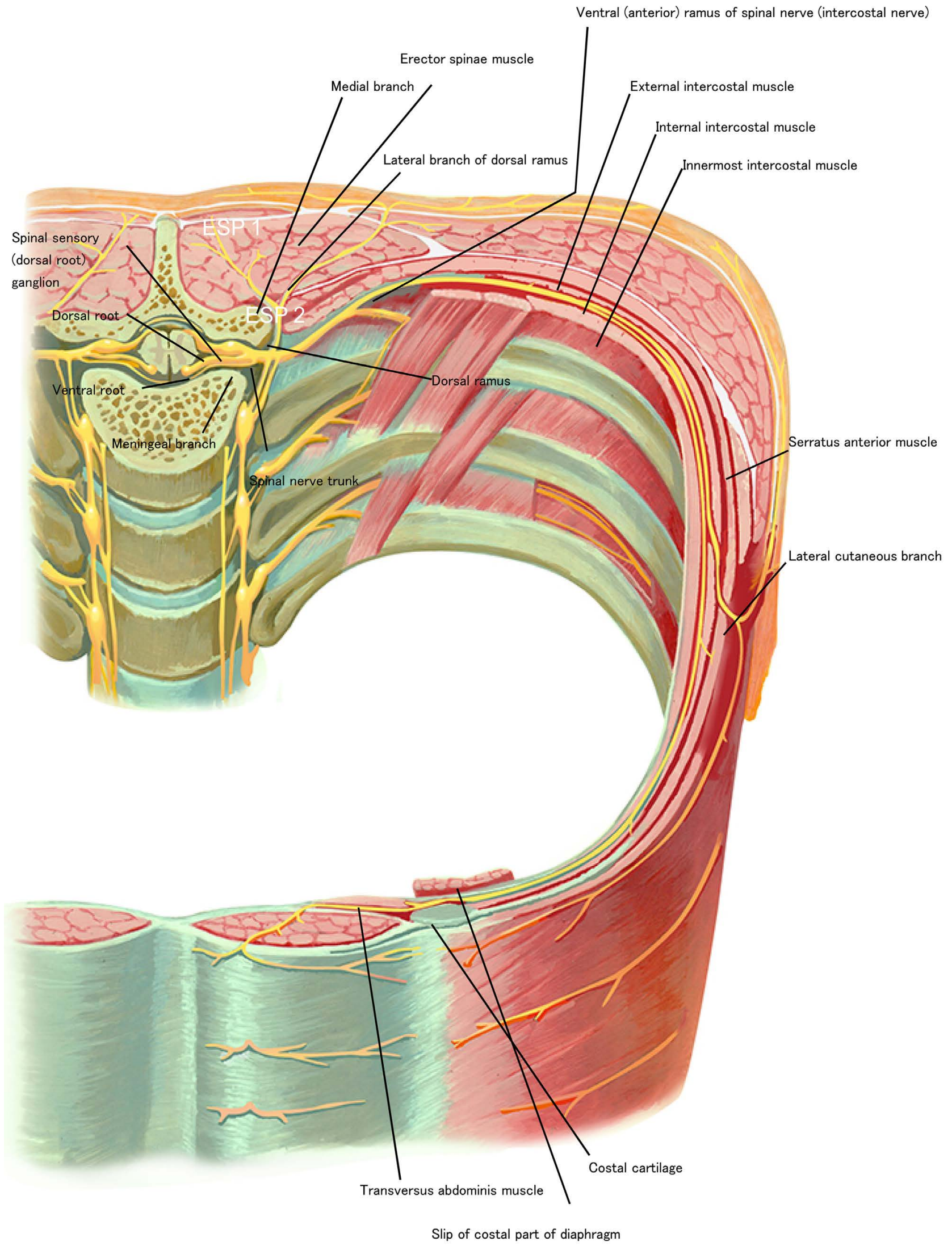

Figure 2. Nomenclature of ESP 1 and ESP 2 (referred to Atlas of Human Anatomy 6th Edition). 


\section{Spread of ESP Block}

The spread of local anesthetic in the ESP block has been investigated in several ways such as by using computed tomography (CT) imaging of cadavers, fluoroscopy, chest radiopgeaphy, and CT imaging of actual patients [1] [13] [14] [15] [16] [17] [18].

These investigations shows that local anesthetics in the ESP block spread tothe upper and lower sides of the interfascial plane between the erector spine muscle and the underlying transverse process. Additionally, the local anesthetic spreads beyond the transverse process to reach the costotransverse junctions, after which it permeates the paravertebral space (Figure 3 ).

\section{Analgesia (Table 1)}

Considering the spread of local anesthetic in the ESP block, it is possible for it to block wider spinal nerves. Therefore, since the first publication, the ESP block has been reported as an effective analgesic for several types of pain and has been used mainly in thoracic surgery. It has also been used in abdominal surgery, nephrectomy, hernia surgery, and hip surgery, among others. Additionally, the ESP block has been used not only acute pain management but also chronic pain management.

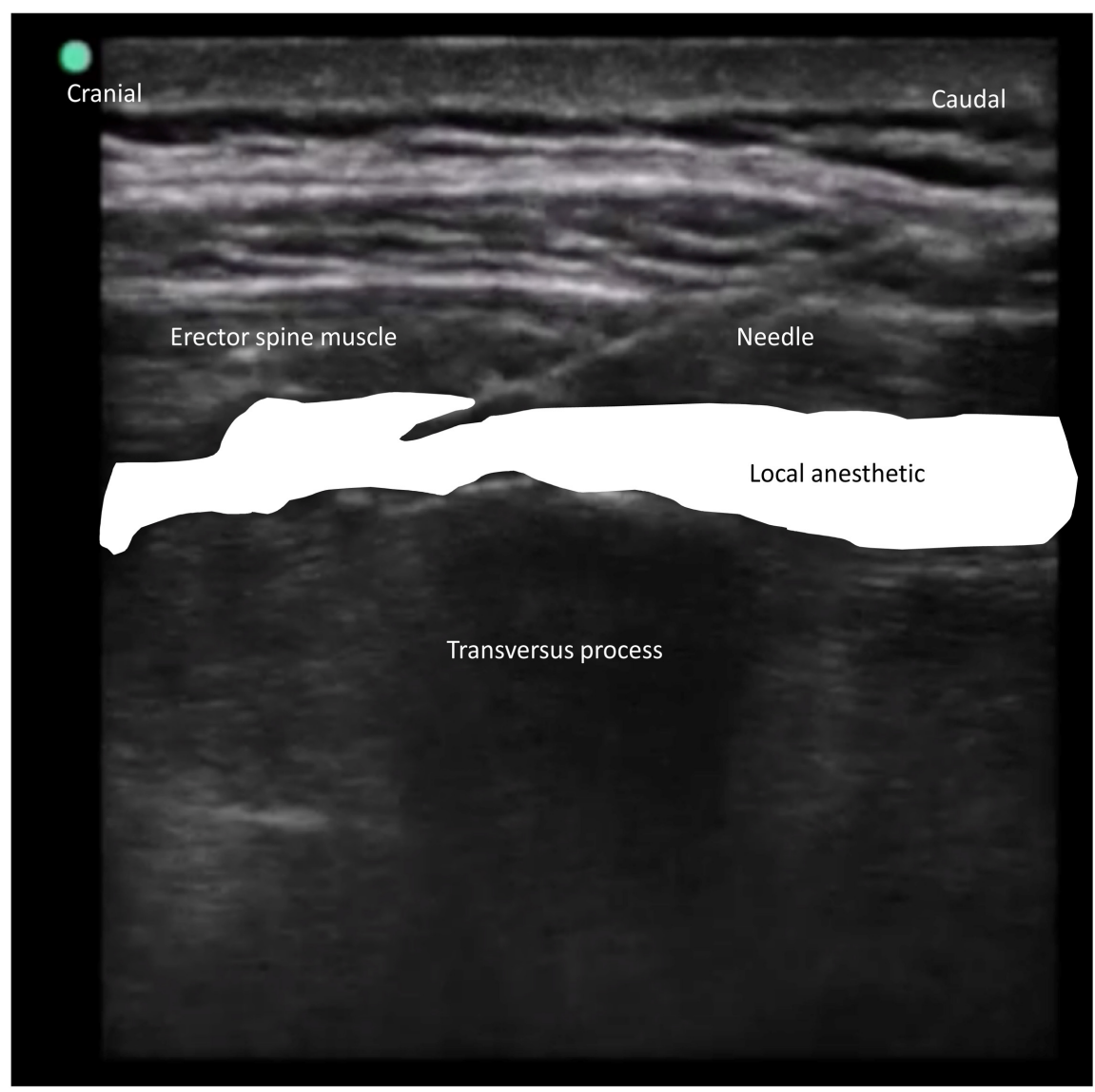

Figure 3. Sagittal view in ESP 2 block. 
Table 1. Indications and spread of local anesthetic in ESP 2 block.

\begin{tabular}{ccc}
\hline Level & Pain & Volumelevel $^{-1}[\mathrm{ml}]$ \\
\hline & Chronic shoulder pain & \\
Mastectomy & $3.1(2.5-6.6)$ \\
Video assisted thoracoscopy & Open thoracic surgery & \\
Chronic thoracic pain & Pneumothorax surgery \\
Abdominoplasty & \\
& Bariatric surgery & $3.7(2.5-6)$ \\
Lentral hernia repair & Open cystoprostatectomy \\
& Nephrectomy & 6.0 \\
& Hip surgery & \\
\hline
\end{tabular}

We do not know the appropriate local anesthetic concentration and volume to be used for ESP block. Considering the past case report, the concentration of the local anesthetic concentration of ropivacaine was $0.5 \%$, that of revobupivacaine was $0.25 \%$ and $0.375 \%$ while bupivacaine concentration were $0.25 \%$ and $0.5 \%$. Additionally, spread of local anesthetic in upper thoracic surgery (3.1 ml.level $\left.{ }^{-1}\right)$ was more extensive lower thoracic $\left(3.7 \mathrm{ml} \cdot \mathrm{level}^{-1}\right)$ and lumbar surgery $\left(6.0 \mathrm{ml} \cdot \mathrm{level}^{-1}\right)$ [1]-[32]. These data are small and vary widely. By a case, a single bolus of $30 \mathrm{ml}$ in the ESP block was of 9 dermatomes [29].

Further, it is difficult to objectify the duration of analgesia after the ESP block. Most case reports show that additional analgesic after the ESP block is not required [2] [19]. In future, we propose a clear description as for the duration of analgesia after the ESP block.

Several methods (intermittent bolus, continuous infusion and patient controlled analgesia) of continuous infusion in ESP blocks have been reported [3] [15] [18] [19]. It is still uncertain which one is the best due to the small size of the studies.

\section{Complication}

Complications of ESP block have never been reported in 2016 and 2017. Pneumothorax after ESP was reported in 2018 [33]. In the future, complications such as arterial puncture and hematoma except pneumothorax should be considered.

\section{Discussion}

Since the original publication on the ESP block in 2016, this technique has been reported as an effective analgesic for several types of pain. Considering the spread of local anesthetic in the ESP block, it is clearly an effective analgesic for the management of several types of pain.

Local anesthetic concentration was used more than $0.25 \%$ of long-acting local anesthetic and volume was about $3.0 \mathrm{ml} \cdot \mathrm{level}^{-1}$. Duration of analgesia after the ESP is still unknown. Therefore, continuous ESP block may be required for ma- 
jor interventions.

In this review, with the exception of a few case reports, there were no RCTs and no observational or other comparative studies. Future research should compare the ESP block to the paravertebral or other nerve blocks. Additionally, the rare of complications and efficacy of analgesic for the ESP block should be investigated.

\section{Conclusion}

Since the original publication on the erector spinae plane (ESP) block in 2016, the report of the ESP block has evolved significantly in the last few years. Currently, it is understood that the ESP block is an effective analgesic for thoracic and abdominal sites in all ages. However, there were no cohort studies or randomized controlled trials in 2016 and 2017. Future research on ESP block is required.

\section{Conflict of Interest}

There are no conflicts of interest.

\section{References}

[1] Forero, M., et al. (2016) The Erector Spinae Plane Block: A Novel Analgesic Technique in Thoracic Neuropathic Pain. Regional Anesthesia and Pain Medicine, 41, 621-627. https://doi.org/10.1097/AAP.0000000000000451

[2] Ueshima, H. and Otake, H. (2018) Clinical Experiences of Erector Spinae Plane Block for Children. Journal of Clinical Anesthesia, 44, 41.

https://doi.org/10.1016/j.jclinane.2017.10.021

[3] De la Cuadra-Fontaine, J.C., Concha, M., Vuletin, F. and Arancibia, H. (2018) Continuous Erector Spinae Plane Block for Thoracicsurgery in a Pediatric Patient. Pediatric Anesthesia, 28, 74-75. https://doi.org/10.1111/pan.13277

[4] Hernandez, M.A., Palazzi, L., Lapalma, J., Forero, M. and Chin, K.J. (2018) Erector Spinae Plane Block for Surgery of the Posterior Thoracic Wall in a Pediatric Patient. Regional Anesthesia and Pain Medicine, 43, 217-219.

[5] Munoz, F., et al. (2017) Erector Spinae Plane Block for Postoperative Analgesia in Pediatric Oncological Thoracic Surgery. Canadian Journal of AnesthesialJournal canadien d anesthésie, 64, 880-882. https://doi.org/10.1007/s12630-017-0894-0

[6] Aksu, C. and Y. Gurkan, (2018) Ultrasound Guided Erector Spinae Block for Postoperative Analgesia in Pediatric Nephrectomy Surgeries. Journal of Clinical Anesthesia, 45, 35-36. https://doi.org/10.1016/j.jclinane.2017.12.021

[7] Willard, F.H., Vleeming, A., Schuenke, M.D., Danneels, L. and Schleip, R. (2012) The Thoracolumbar Fascia: Anatomy, Function and Clinical Considerations. Jour nal of Anatomy, 221, 507-536. https://doi.org/10.1111/j.1469-7580.2012.01511.x

[8] Ahiskalioglu, A., Alici, H.A. and Ari, M.A. (2017) Ultrasound Guided Low Thoracic erector Spinaeplane Block for Management of Acute Herpes Zoster. Journal of Clinical Anesthesia, 45, 60-61. https://doi.org/10.1016/j.jclinane.2017.12.018

[9] Chin, K.J., Adhikary, S. and Forero, M. (2017) Is the Erector Spinae Plane (ESP) Block a Sheathblock? A Reply. Anaesthesia, 72, 916-917.

https://doi.org/10.1111/anae.13926 
[10] Hamilton, D.L. and Manickam, B.P. (2017) Is the Erector Spinae Plane (ESP) Block a Sheath Block? Anaesthesia, 72, 915-916. https://doi.org/10.1111/anae.13912

[11] Murouchi, T. (2017) Consideration of Block Nomenclature: Erector Spinae Plane Block or Retrolaminar Block? Regional Anesthesia and Pain Medicine, 42, 124. https://doi.org/10.1097/AAP.0000000000000525

[12] Ueshima, H. and Otake, H. (2017) Similarities between the Retrolaminar and Erector Spinae Plane Blocks. Regional Anesthesia and Pain Medicine, 42, 123-124. https://doi.org/10.1097/AAP.0000000000000526

[13] Chin, K.J., et al. (2017) The Analgesic Efficacy of Pre-Operative Bilateral Erector Spinae Plane (ESP) Blocks in Patients Having Ventral Hernia Repair. Anaesthesia, 72, 452-460. https://doi.org/10.1111/anae.13814

[14] Ueshima, H. and Hiroshi, O. (2017) Spread of Local Anesthetic Solution in the Erector Spinae Plane Block. Journal of Clinical Anesthesia, 45, 23. https://doi.org/10.1016/j.jclinane.2017.12.007

[15] Ashok, J., Priyanka, J. and Neelam, S. (2017) The Erector Spinae Block for Postoperative Analgesia in Abdominoplasty-A Case Report. BAOJ Anesthesia, 1, 1.

[16] Forero, M., et al. (2017) Erector Spinae Plane Block for the Management of Chronic Shoulder Pain: A Case Report. Canadian Journal of Anesthesia, 65, 288-293.

[17] Hamilton, D.L. and Manickam, B. (2017) The Erector Spinae Plane Block. Regional Anesthesia and Pain Medicine, 42, 276. https://doi.org/10.1097/AAP.0000000000000565

[18] Chin, K.J., Malhas, L. and Perlas, A. (2017) The Erector Spinae Plane Block Provides Visceral Abdominal Analgesia in Bariatric Surgery: A Report of 3 Cases. Regional Anesthesia and Pain Medicine, 42, 372-376. https://doi.org/10.1097/AAP.0000000000000581

[19] Tanaka, N., Ueshima, H. and Otake, H. (2017) Erector Spinae Plane Block for Combined Lovectomy and Radical Mastectomys. Journal of Clinical Anesthesia, 45, 27-28. https://doi.org/10.1016/j.jclinane.2017.12.012

[20] Ohgoshi, Y., Ikeda, T. and Kurahashi, K. (2018) Continuous Erector Spinae Plane Block Provides Effective Perioperative Analgesia for Breast Reconstruction Using Tissue Expanders: A Report of Two Cases. Journal of Clinical Anesthesia, 44, 1-2. https://doi.org/10.1016/j.jclinane.2017.10.007

[21] Veiga, M., Costa, D. and Brazao, I. (2018) Erector Spinae Plane Block for Radical Mastectomy: Anew Indication? Revista Española de Anestesiología y Reanimación, 65, 112-115. https://doi.org/10.1016/j.redar.2017.08.004

[22] Bonvicini, D., et al. (2018) Bilateral Ultrasound-Guided Erector Spinae Plane Blocks in Breast Cancer and Reconstruction Surgery. Journal of Clinical Anesthesia, 44, 3-4. https://doi.org/10.1016/j.jclinane.2017.10.006

[23] Bonvicini, D., Giacomazzi, A. and Pizzirani, E. (2017) Use of the Ultrasound-Guided Erectorspinae Plane Block in Breast Surgery. Minerva Anestesiologica, 83, 1111.

[24] Scimia, P., et al. (2017) The Ultrasound-Guided Continuous Erector Spinae Plane Block for Postoperative Analgesia in Video-Assisted Thoracoscopic Lobectomy. Regional Anesthesia and Pain Medicine, 42, 537. https://doi.org/10.1097/AAP.0000000000000616

[25] Forero, M., et al. (2017) Continuous Erector Spinae Plane Block for Rescue Analgesia in Thoracotomy after Epidural Failure: A Case Report. A \& A Case Reports, 8 , 254-256. https://doi.org/10.1213/XAA.0000000000000478

[26] Ahiskalioglu, A., et al. (2017) Continuous Ultrasound Guided Erector Spinae Plane 
Block for the Management of Chronic Pain. Anaesthesia Critical Care \& Pain Medicine.

[27] Ueshima, H. and Otake, H. (2017) Erector Spinae Plane Block Provides Effective Pain Management during Pneumothorax Surgery. Journal of Clinical Anesthesia, 40, 74. https://doi.org/10.1016/j.jclinane.2017.04.016

[28] Leyva, F.M., et al. (2017) Continuous Erector Spinae Plane (ESP) Block for Postoperative Analgesia after Minimally Invasive Mitral Valve Surgery. Journal of Cardiothoracic and Vascular Anesthesia, in press. https://doi.org/10.1053/j.jvca.2017.12.020

[29] Forero, M., et al. (2017) Erector Spinae Plane (ESP) Block in the Management of Post Thoracotomy Pain Syndrome: A Case Series. Scandinavian Journal of Pain, 17, 325-329. https://doi.org/10.1016/j.sjpain.2017.08.013

[30] Restrepo-Garces, C.E., et al. (2017) Bilateral Continuous Erector Spinae Plane Block Contributes to Effective Postoperative Analgesia after Major Open Abdominal Surgery: A Case Report. A \& A Case Reports, 9, 319-321. https://doi.org/10.1213/XAA.0000000000000605

[31] Ueshima, H. and Otake, H. (2017) Clinical Experiences of Ultrasound-Guided Erector Spinae Plane Block for Thoracic Vertebra Surgery. Journal of Clinical Anesthesia, 38, 137. https://doi.org/10.1016/j.jclinane.2016.12.028

[32] Tulgar, S. and Senturk, O. (2018) Ultrasound Guided Erector Spinae Plane Block at L-4 Transverse Process Level Provides Effective Postoperative Analgesia for Total Hip Arthroplasty. Journal of Clinical Anesthesia, 44, 68. https://doi.org/10.1016/j.jclinane.2017.11.006

[33] Ueshima, H. (2018) Pneumothorax after the Erector Spinae Plane Block. Journal of Clinical Anesthesia, 48, 12. https://doi.org/10.1016/j.jclinane.2018.04.009 